DOI: $10.2478 /$ v.10169-010-0018-5

\title{
FRACTAL DIMENSION FOR THE CHARACTERIZATION OF THE POROSITY OF ASPHALT CONCRETES
}

\author{
G. LEONARDI ${ }^{1}$
}

\begin{abstract}
In the design of asphalt mixtures for paving, the choice of components has a remarkable importance, as requirements of quality and durability must be assured in use, guaranteeing adequate standards of safety and comfort.

In this paper, an approach of analysis on the aggregate materials using fractal geometry is proposed. Following an analytical and an experimental approach, it was possible to find a correlation between characteristics of the asphalt concrete (specific gravity and porosity) and the fractal dimension of the aggregate mixtures.

The studies revealed that this approach allows to draw the optimal fractal dimension and, consequently, it can be used to choose an appropriate aggregate gradation for the specific application; once the appropriate initial physical parameters are finalized.

This fractal approach could be employed for predicting the porosity of mixed asphalt concretes, given as input the fractal characteristics of the aggregate mixtures of the concrete materials.
\end{abstract}

Key words: fractal geometry, porosity, mixture, aggregate, concrete, pavement.

\section{InTRODUCTION}

Some researchers maintain since some time that fractal geometry can contribute to the description and interpretation of the microstructural complexity of hydrated cements. The applications deal with both the solid phases and the complementary porosity .[1].

Winslow [2] recognises the fractal character of the surfaces of cement pastes. Livingston [3] guesses the fractality in the process of nucleation and growth of tricalcium silicate. Lange et al. [4] use the concept of fractal dimensions to describe the structure of pores defined through image analysis, while Panagouli and Kokkalis .[5] applies it to calculate the skid resistance of roads pavements.

A fractal character was recognised on materials similar to cement from a porosimetric point of view such as limes, soils, rocks, and ceramics [6, 7]. Huang et al. [8] illustrated the correlation between fractal dimension and porosity of porous solid.

\footnotetext{
1 Associate Professor, DIMET - University of Reggio Calabria, e-mail: leonardi.giovanni@gmail.com - giovanni.leonardi@unirc.it
} 
The aim of this paper is to demonstrate the applicability of fractals geometry to characterize the porosity of mixed asphalt concretes.

\section{Fractal GeOMEtry}

Fractal geometry is a field of modern mathematics, born at the beginning of 20th century, to describe irregular geometrical forms.

However, this new field of geometry became methodologically important for applied research only after Benoit B. Mandelbrot's works [9, 10], where he pointed out some peculiar geometrical properties of certain objects, as the coasts of continents, the branches of trees, or the surfaces of clouds.

Mandelbrot coined the word fractal (in Latin, fractus means irregular) for these complex forms in order to express that their peculiarity is a non-integer dimension that is fractal [11-13].

The simplest fractals are self-similar fractals constructed by repeating a pattern or generator onto a starting object or initiator. The initiator determines the dimensionality, whereas the generator defines the overall symmetry of the object and produces features at different length scales [6].

The generator pattern is repeated $n$ times and can result in either accretion or reduction of the initiator. The final fractal object contains a range of lengths, $r=1 / b^{i}$, where $b$ is a scaling factor and $i=1,2,3, \ldots, n$. Examples of fractal objects generated by this process are shown in the following figures. In the Cantor bar (Fig. 1), the initiator is a solid line and the generator is a broken line. The initial solid line is divided into three equal segments and the middle third is removed (reductive algorithm). The process is then repeated for each of the remaining solid segments. The initiator for the Koch curve (Fig. 2) is also a solid line that is divided into three segments, but the middle segment is now replaced by two segments of the same length as the outer ones (accretive algorithm). The Sierpinski carpet and its three-dimensional counterpart the Menger sponge (Fig. 3) are produced by removal of squares or cubes from the initiator which is a plane or a cube, respectively. For example, in the Menger sponge, a cube of unit length is partitioned into 27 smaller cubes and seven cubes are removed. This process is then repeated for each of the remaining solid cubes.

The above mentioned fractals are deterministic since the same operation is repeated at all scales. Randomness can be introduced in these constructions in different ways to produce statistical fractals.

\subsection{Fractal Dimension}

The fundamental equation applying to all fractals is the number-size relationship .[9, 14]: 


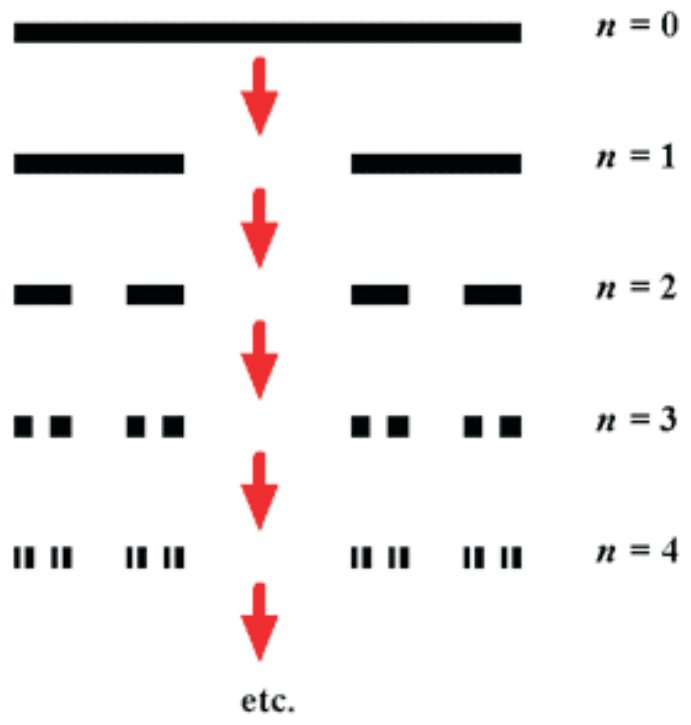

Fig. 1. Cantor bar.

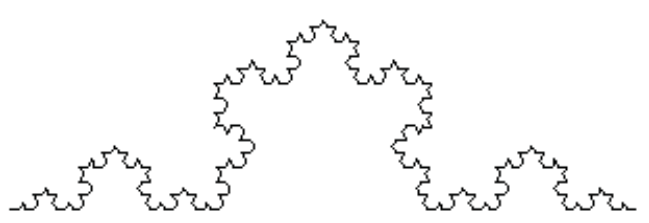

Fig. 2. Koch curve.

$$
N(r)=k \cdot\left(\frac{1}{r}\right)^{D}=k \cdot r^{-D}
$$

where $N(r)$ is the number of elements of length equal to $r, K$ is the number of initiators of unit length and Dis the fractal dimension. Or, equivalently:

$$
D=\frac{[\log N(r)-\log k]}{\log (1 / r)} .
$$

The concept of fractal dimension admits multiples definitions. All of they are seemed ????, and constitute generalizations of classical concept of integer dimension, which 


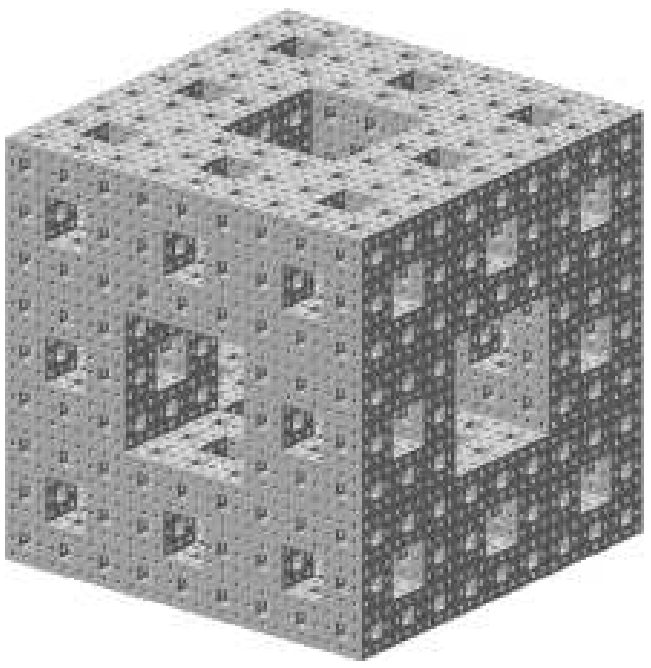

Fig. 3. Menger sponge.

recovers. Perhaps the most intuitive and simple to understand is homothetic dimension or self-similarity dimension.

A geometrical object is said self-similar if it can be arbitrarily decomposed into small parts, which are all copies of the whole structure; those sub-components are obtained by means of a homothetic transformation.

Consider a set $\mathrm{G}$ of points whose positions are given by the vectors:

$$
\vec{P}_{i}=\left(x_{1_{i}}, \ldots \ldots \ldots, x_{E_{i}}\right),
$$

in a $E$-dimensional Euclidean space, applying a reduction (or a dilatation) characterized by a scale ratio $r$, the set $\mathrm{G}$ becomes the set $r \mathrm{G}$, where the points are positioned by:

$$
r \vec{P}_{i}=\left(r x_{1_{i}}, \ldots \ldots, r x_{E_{i}}\right) .
$$

A limited set $\mathrm{G}$ is said "self-similar" if it is made by the union of $\mathrm{N}$ distinct sub-sets (with void intersection) which can be superimposed by means of rototranslactions to $r$ G. The number of copies $N$ in which a $D$-dimensional self-similar object can be divided by means of a scale factor $r$ is given by the relation:

$$
N \propto r^{-D},
$$

where $D$ is the homothetic dimension or self-similarity dimension ...[10]. 


\subsection{AgGregates Size Distribution AND FRACTAL Dimension}

In the last years, soils and aggregate size distributions were characterized by fractal dimension $(D$, i.e., slope of the log of cumulative aggregate number as a function of the log of aggregate size) and several authors observed self-similarity in different organization levels of soils and mineral aggregates [15-18]. So, fractal geometry was used to model hydraulic proprieties by Giménez et al. [6] and Atzeni et al. [1], to study the porosity by Atzeni et al., [7], Huang et al., [8], Lange et al., [4] and Perrier et al., [19] and to model the surface characteristics of cement pastes and concretes by Kokkalis and Panagouli [20] and Winslow [2].

To study the fractal proprieties of aggregates, it is necessary to analyse the mixtures in volumetric terms, as also suggested in "Code de bonne pratique pour la formulation des enrobés bitumineux" [21] and in Cominsky et al. [22]. To express the grain-size distribution curve in terms of volume, we need to calculate the density of the various size classes of the aggregates. After the estimation of the mean volume of the single element, we can obtain a representation in terms of cumulated number.

In order to reach this result, it is necessary to make some simple considerations about the form of the single grain of a mixture of aggregates.

The single grain, not too irregular, can be assumed to be an ellipsoid having width $b$, length $a=\mathrm{k} \cdot b$ and thickness $s=b / \mathrm{k}$, as shown in Fig. 4:
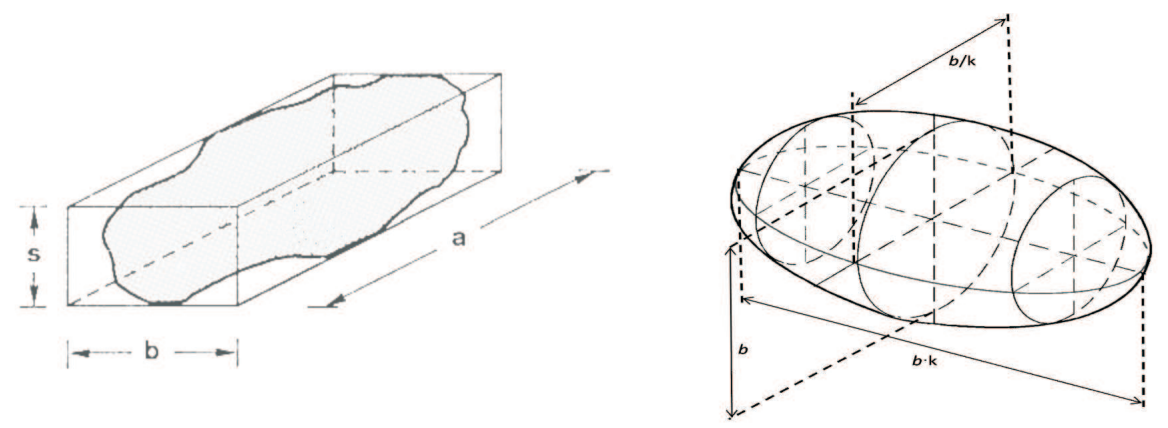

Fig. 4. Grain dimensions.

The volume of this ellipsoid is:

$$
\frac{\pi}{6} \cdot b \cdot a \cdot s=\frac{\pi}{6} \cdot(k \cdot b) \cdot b \cdot(b / k)=\frac{\pi}{6} \cdot b^{3}
$$

Therefore, it is evident that the volume of each grain is equivalent to the volume of a sphere with diameter $b$. 
There exists a correlation between the coefficient $k$ and the ratio between the actual volume of the grain and the volume of the smallest sphere which holds it. This correlation is given by:

$$
\frac{\frac{\pi}{6} \cdot a \cdot b \cdot s}{\frac{\pi}{6} \cdot a^{3}}=\frac{\frac{\pi}{6} \cdot(k \cdot b) \cdot b \cdot(b / k)}{\frac{\pi}{6} \cdot(k \cdot b)^{3}}=k^{-3} .
$$

The typical $k$ values for aggregate grains of common use are between 1.35 and 1.5.

As a consequence, assuming that the grains making up the mixture have all an ellipsoidal form, all the elements with width $b<D$ will pass through an aperture size of diameter $D$, after a sufficient time interval.

To obtain the cumulated number of elements it is necessary to calculate the density of the fractions which pass through the sieve with an aperture size $D$ and stopped by the lower one of aperture size $d$.

Known the mass $M$ and the density $\gamma$ and assuming, in first approximation, that the mean volume of the single grain is equal to $(\pi / 6) \cdot b^{3}$, where $b$ is the diameter of the equivalent sphere, the number of grains of the passing fraction is given by:

$$
N=\frac{M}{\gamma \cdot b^{3} \cdot\left(\frac{\pi}{6}\right)} .
$$

Moreover, the cumulated number of grains, with mean dimensions greater or equal to $b$, is given by the sum:

$$
N_{c}\left(b_{i}>d\right)=\sum_{i} \frac{M_{i}}{\gamma_{i} \cdot b_{i}^{3} \cdot\left(\frac{\pi}{6}\right)} .
$$

The cumulated number of the elements, as the dimensions change, can be evaluated as illustrated in [18], they found the following relation:

$$
N_{c}(d)=B \cdot d^{-D},
$$

the eq. (2.10) clearly shows the fractal character of the grain-size distribution curves, expressed in terms of cumulated number of elements.

In fact, remembering the definitions of fractal dimension, we can write:

$$
N \propto r^{-D} .
$$

It follows from eq. (2.10) that the graph of $\log \left(N_{c}\right)$ vs. $\log (d)$ should be approximately a straight line with slope equal to $-D$. 
Now we want to extend this fractal representation to investigate the existing correlation between porosity characteristic of a composite materials constituted by mineral aggregates glued together with a binder like the asphalt concrete (e. g. Hot Mix Asphalt Concrete HMA) and the fractal dimension of the mixture.

It has long been established that gradation of the aggregate is one of the factors that must be carefully considered in the design of asphalt paving mixtures. In fact, the extent to which an HMA mixture can be compacted is strongly related to aggregate gradation [23]. Aggregate gradation is the size distribution of the aggregate particles, including the amount of material passing the $75-\mathrm{mm}$ sieve (dust content).

Goode and Lufsey [23] demonstrated that an aggregate having a gradation that produces a straight line on a 0.45 in a power gradation graph will have the maximum achievable density, and subsequently the lowest air void content and the lowest voids in the mineral aggregate (VMA) in an HMA mixture.

However, Huber and Shuler [24] note that significant confusion exists concerning different methods used to draw aggregate gradation "maximum" density lines. Closely related to maximum density lines, and also in debate, is the definition of nominal aggregate maximum size.

The purpose of this paper is to demonstrate that an aggregate mixture having a gradation that produces an optimal fractal dimension will have the maximum achievable density, and subsequently the lowest air voids in an HMA.

\section{Materials AND MEthodology}

In the study and use of asphalt concretes for road pavements, the main problem is the choice and dosage of the components (mineral aggregates and asphalt) in order to obtain a mix with the appropriate performance and durability characteristics, in relation to the use and environment the infrastructure is exposed to.

Given the many variables to be taken into account, it is obviously difficult to assess the quality of an asphalt mixture, during the design phase. Therefore the necessity to analyze new methodologies is evident which can strongly support the road planner in order to optimize the comprehensive outcome of his design.

From this point of view, it can be particularly interesting to investigate new analytic approach to explain the influence of the characteristics of aggregate materials, in particular the volumetric distribution, on the proprieties of the HMA.

The first step of this approach is represented by the characterization of the mixtures by means of fractal dimension.

Therefore, if the computation of the approximate volume of the single grain is possible and the volume mass of the single class is supposed to be known and being careful to evaluate $d_{i}$ distribution of the characteristic dimensions of the grains within each class; it is possible to evaluate the fractal dimension of mixture starting from a gradation analysis. 
We evaluate the mean dimension of the grains of a gradation class, passing through the sieve $d_{y}$ and stop by the sieve $d_{x}$, by means of the formula [21]:

$$
G_{r i f}=\frac{\ln d_{y}-\ln d_{x}}{\frac{1}{d_{x}}-\frac{1}{d_{y}}} .
$$

After the characteristic points $\left(N_{c}\right.$ and $\left.G_{r i f}\right)$ of the various classes of the mixture are obtained, it is possible to single out the corresponding regression straight line on a logarithm plane, whose slope represents the fractal dimension of the considered mixture.

Now, the aim of the proposed research is to analyse a possible correlation between the fractal dimension of the aggregates mixture and a physical characteristic of the asphalt concrete (i. e. the porosity). To reach this aim firstly we studied 11 mineral aggregate mixtures (Fig. 5).

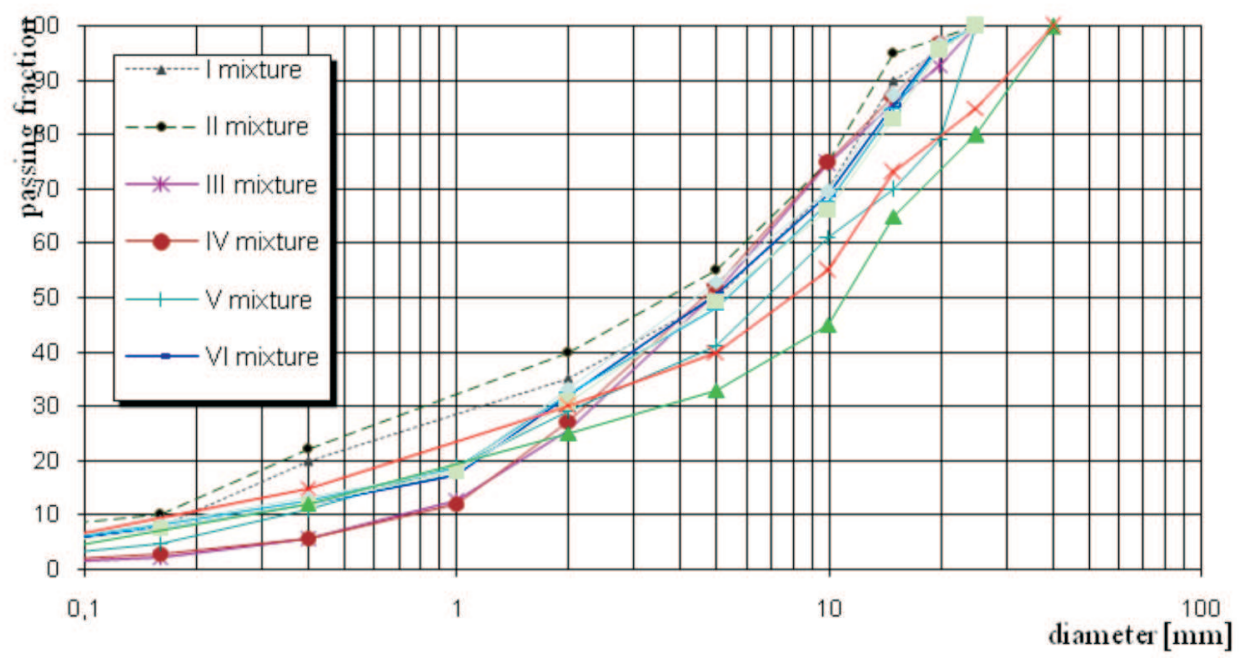

Fig. 5. Gradation of the considered eleven aggregate mixtures.

According to eq. 2.9 and eq. 3.1 the cumulated number of the elements $N_{i}$ and the reference dimension for the different gradation class $G_{r i f}$ were calculated.

The obtained data for the 11 mixtures were plotted as illustrated in Fig. 6, where slope of the linear regression was -2.84 corresponding to a fractal dimension equal to 2.84 for the mixture number 1 (Table 1 ).

The same approach allowed us to calculate the fractal dimension of all the 11 mixtures.

The 11 aggregate mixtures were used to form 11 different hot mixed asphalt concretes (HMA) in order to investigate the researched correlation. 


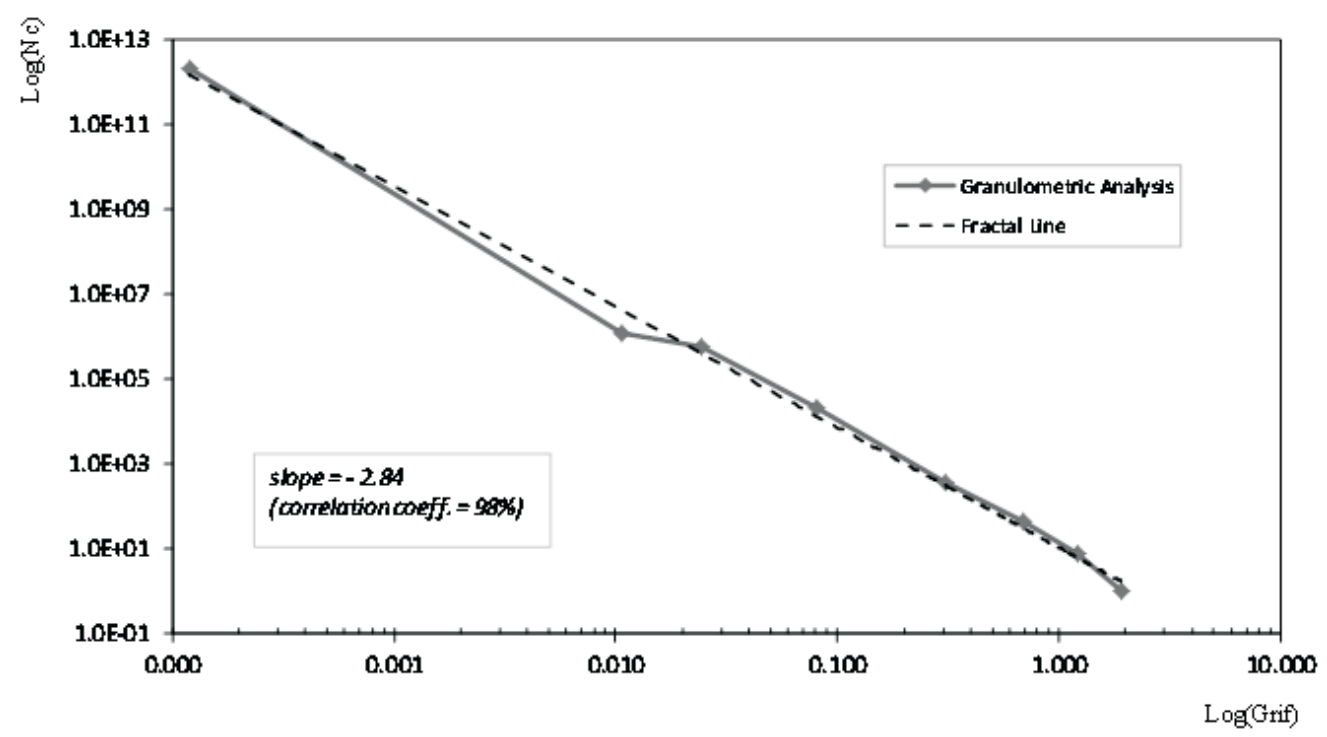

Fig. 6. Example of interpolation for the determination of the fractal dimension.

Table 1

Aggregate density of different size classes (mixture n. 1).

\begin{tabular}{|c|c|c|c|}
\hline Aggregate size $[\mathrm{mm}]$ & Density, $\left[\mathrm{kg} \cdot \mathrm{m}^{-3}\right]$ & $\begin{array}{c}\mathrm{G}_{\text {rif }} \\
{[\mathrm{mm}]}\end{array}$ & $\mathrm{N}_{i}$ \\
\hline$<1.5$ & 2720 & 1.9156 & $1.0 \mathrm{E}+00$ \\
\hline $1.5-1.0$ & 2730 & 1.2164 & $7.8 \mathrm{E}+00$ \\
\hline $1.0-0.5$ & 2720 & 0.6931 & $4.2 \mathrm{E}+01$ \\
\hline $0.5-0.2$ & 2700 & 0.3054 & $3.7 \mathrm{E}+02$ \\
\hline $0.2-0.04$ & 2680 & 0.0805 & $2.1 \mathrm{E}+04$ \\
\hline $0.04-0.016$ & 2700 & 0.0244 & $5.8 \mathrm{E}+05$ \\
\hline $0.016-0.0075$ & 2700 & 0.0107 & $1.2 \mathrm{E}+06$ \\
\hline$<0.0075$ & 3300 & 0.00012 & $2.1 \mathrm{E}+12$ \\
\hline
\end{tabular}

The HMA was produced using the same type and quantity $(4.30 \%)$ of binder (bitumen).

\section{RESUlTS AND DISCUSSION}

Table 2 summarizes the estimated fractal dimension, porosity and specific gravity of the 11 HMA concretes.

We have found that the values of the porosity in function of the fractal number point out the existence of an optimal value of the fractal dimension in connection with 
the aim of maximum condensation, whose value can be estimated in 2.87. For greater values we can image an opposite trend of the variable (Fig. 7).

As expected, a similar behavior was obtained for the definition of specific gravity fractal dimension. In fact, also in this case an optimal fractal dimension can be found.

Summary of fractal dimension and porosity for the eleven HMA.

Table 2

\begin{tabular}{|c|c|c|c|}
\hline HMA & $\begin{array}{c}\text { Fractal } \\
\text { dimension }\end{array}$ & $\begin{array}{c}\text { porosity } \\
{[\%]}\end{array}$ & $\begin{array}{c}\text { specific gravity } \gamma_{c} \\
{\left[\mathrm{~kg} / \mathrm{m}^{3}\right]}\end{array}$ \\
\hline 1 & 2.839 & 5.59 & 2400 \\
\hline 2 & 2.896 & 5.31 & 2410 \\
\hline 3 & 2.772 & 11.90 & 2241 \\
\hline 4 & 2.755 & 12.05 & 2217 \\
\hline 5 & 2.772 & 9.00 & 2310 \\
\hline 6 & 2.868 & 6.54 & 2342 \\
\hline 7 & 2.870 & 5.23 & 2361 \\
\hline 8 & 2.889 & 6.21 & 2336 \\
\hline 9 & 2.850 & 6.82 & 2329 \\
\hline 10 & 2.811 & 7.10 & 2370 \\
\hline 11 & 2.877 & 5.90 & 2400 \\
\hline
\end{tabular}

The optimal values of the fractal dimension in connection with these two physical quantities are almost coincident (2.866 and 2.875) and they are very near to the theoretical limit pertinent to a condensation compared to that of solid, which is obtained when the fractal dimension is equal to 3 .

For simplicity, the obtained results are plotted in the following figures.

\section{Conclusions}

The premise to the study suggested the use of fractal geometry for the characterization of the proprieties of road asphalt concrete finds, and the results confirm the good fit and reliability of the approach.

The fractal dimension represents an appropriate parameter in order to determine an adequate grain-size selection which can guarantee the achievement of certain values of porosity and specific gravity.

In fact it is possible to determine, in equal conditions, a relationship between fractal dimension and the concrete characteristics.

So that, for example, from a determined porosity value, chosen for a particular type of road surface; it is possible to go back to the relative fractal dimension and consequently to design easily the aggregates mixture. 


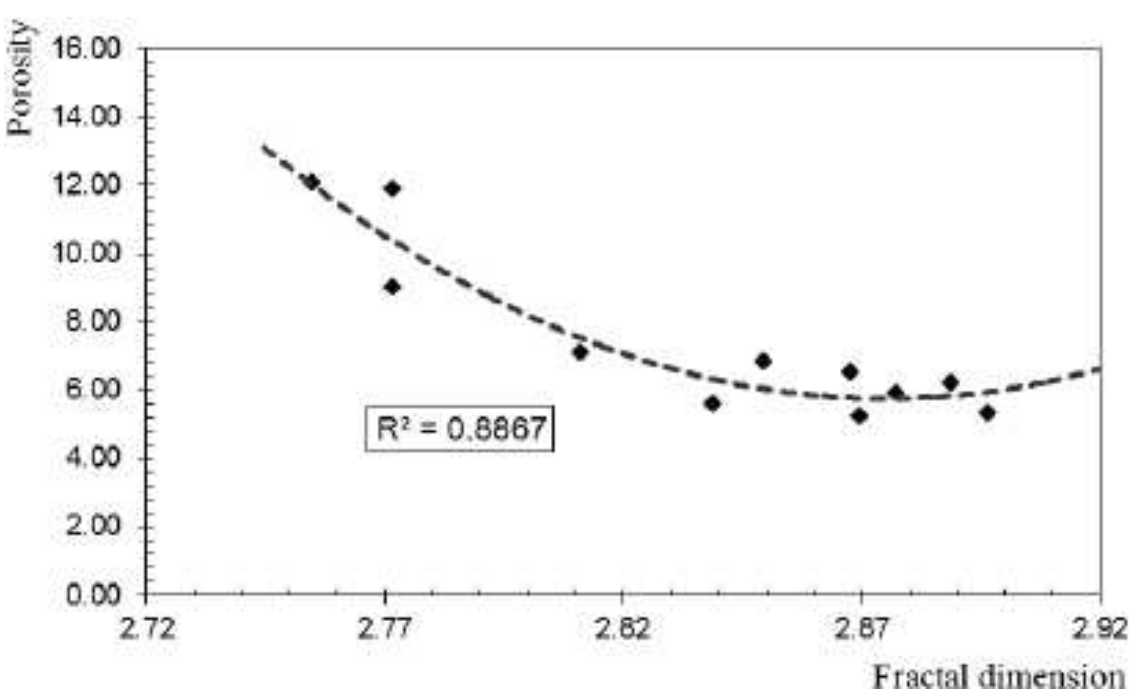

Fig. 7. Relations between porosity and fractal dimension.

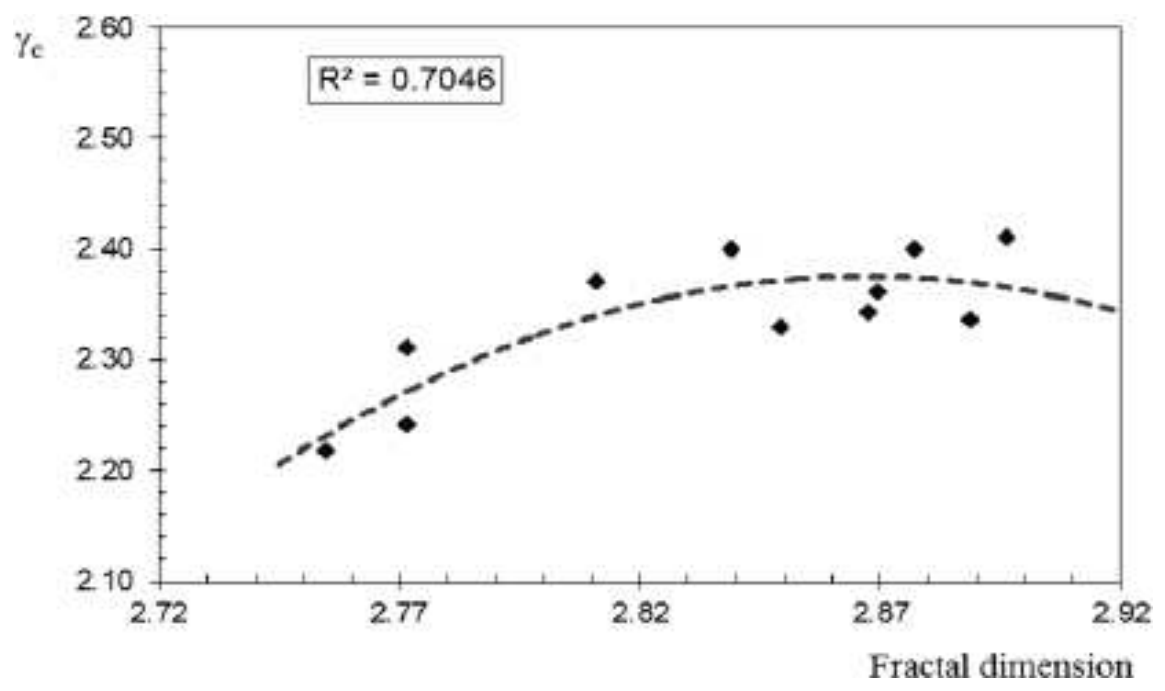

Fig. 8. Relations between specific gravity and fractal dimension. 
The identification of fractal properties and an effective correlation with porosity could prove useful for improving the understanding, characterization and control of the properties of these materials.

\section{REFERENCES}

1. C. Atzeni, et al., A geometrical fractal model for the porosity and permeability of hydraulic cement pastes, Construction and Building Materials, 2010.

2. D.N. Winslow, The fractal nature of the surface of cement paste, Cement and Concrete Research, 15, pp. 817-824, 1985.

3. R.A. Livingston, Fractal nucleation and growth model for the hydration of tricalcium silicate, Cement and Concrete Research, 30, pp. 1853-1860, 2000.

4. D.A. LANGE, et al., Image analysis techniques for characterization of pore structure of cement-based materials, Cement and Concrete Research, 24, pp. 841-853, 1994.

5. O. Panagoul, A. KoKKalis, Skid resistance and fractal structure of pavement surface, Chaos, Solitons \& Fractals, 9, pp. 493-505, 1998.

6. D. GimÉNEZ, et al., Fractal models for predicting soil hydraulic properties: a review, Engineering Geology, 48, pp. 161-183, 1997.

7. C. Atzeni,et al., A fractal model of the porous microstructure of earth-based materials, Construction and Building Materials, 22, pp. 1607-1613, 2008.

8. S. HuAng,et al., Correlations and characterization of porous solids by fractal dimension and porosity, Physica A: Statistical Mechanics and its Applications, 274, pp. 419-432, 1999.

9. B. Mandelbrot, The fractal geometry of nature: Wh Freeman, 1982.

10. B. Mandelbrot, A. Blumen, Fractal Geometry: What is it, and What Does it do?[and Discussion], Proceedings of the Royal Society of London. Series A, Mathematical and Physical Sciences, 423, pp. 3-16, 1989.

11. H. Peitgen,et al., Bausteine des Chaos: Fraktale: Springer Verl.; Klett-Cotta, 1992.

12. H. Peitgen, P. Richter, The beauty of fractals: Springer-Verlag New York, 1986.

13. H. Peitgen,et al., The science of fractal images: Springer New York etc., 1988.

14. J. Feder, P. BaK, Fractals, Physics Today, 42, p. 90, 1989.

15. A. Anderson, A. McBratney, Soil aggregates as mass fractals, Australian Journal of Soil Research, 33, pp. 757-772, 1995.

16. F. BARTOLI,et al., Structure and self-similarity in silty and sandy soils: the fractal approach, European Journal of Soil Science, 42, pp. 167-185, 1991.

17. I.M. Young, J.W. CRAWFord, Fractal structure of soil aggregates: its measurement and interpretation : J Soil Sci V42, N2, June 1991, P187-192, International Journal of Rock Mechanics and Mining Sciences \& Geomechanics Abstracts, 28, pp. A336-A336, 1991.

18. M. Rieu, G. Sposito, Fractal fragmentation, soil porosity, and soil water properties: I. Theory, Soil Sci. Soc. Am. J, 55, p. 1, 1991.

19. E. Perrier, et al., Computer construction of fractal soil structures: simulation of their hydraulic and shrinkage properties, International Journal of Rock Mechanics and Mining Sciences \&amp; Geomechanics Abstracts, 33, pp. 259A-260A, 1996.

20. A. Koкkalis, O. Panagoul, Fractal Evaluation of Pavement Skid Resistance Variations. I: Surface Wetting, Chaos, Solitons \& Fractals, 9, pp. 1875-1890, 1998.

21. C.R.R., Code de Bonne Pratique pour la Formulation des Enrobés Bitumineux Denses. Recommandations CRR - R 61/87, ed. Bruxelles, Belgium: Centre de Recherches Routičres, 1987. 
22. R. Cominsky,et al., SHRP-A-407. The Superpave Mix Design Manual for New Construction and Overlays, National Research Council, Washington, DC, 1994.

23. J. Goode, L. Lufsey, A new graphical chart for evaluating aggregate gradations, pp. 176-207, 1962.

24. G. Huber, T. Shuler, Providing Sufficient Void Space for Asphalt Cement: Relationship of Mineral Aggregate Voids and Aggregate Gradation, Effects of Aggregates and Mineral Fillers on Asphalt Mixture Performance, 1992.

Remarks on the paper should be sent to the Editorial Office

no later than March 30, 2011
Received September 01, 2010 revised version December 10, 2010 